\title{
Exploring energy landscapes at the DFTB quantum level using the threshold algorithm : the case of the anionic metal cluster
} $\mathrm{Au}_{20}^{-}$

\author{
Mathias Rapacioli • Johann Christian Schön • Nathalie Tarrat
}

Received: date / Accepted: date

\begin{abstract}
We report the combination of the threshold algorithm with the Density Functional based Tight Binding method (DFTB) allowing for the exploration of complex potential energy surfaces and the evaluation of probability flows between their regions, at the quantum level. This original scheme is used to explore the energy landscape of an anionic 20-atom gold cluster, $\mathrm{Au}_{20}^{-}$. On the basis of the relevant structures, 19 structural groups are highlighted, all of them being variations about the pyramidal shape : (i) distorted pyramids, (ii) pyramids in which the atom of one of the facets has been removed, leaving a hole, and placed at different positions on the cluster and (iii) pyramids on which an atom located at a vertex has been removed and placed on an edge or on a facet. Upper limits of the energies required to connect the basins of the 19 groups on the potential energy surface are evaluated. Moreover, the attractive basins are identified on the basis of the analysis of the probability flows on the landscape. The comparison of the disconnectivity tree with the results of the flux analysis provides a consistent representation of the $\mathrm{Au}_{20}^{-}$basins' proximity. Finally, we show how the new scheme allowed for the identification of counter-intuitive transition pathways.
\end{abstract}

Mathias Rapacioli

Laboratoire de Chimie et Physique Quantiques LCPQ/IRSAMC, UMR5626, Université de Toulouse (UPS) and CNRS, Toulouse, France

E-mail: mathias.rapacioli@irsamc.ups-tlse.fr

Johann Christian Schön

Max Planck Institute for Solid State Research, Heisenbergstr. 1, 70569 Stuttgart, Germany E-mail: C.Schoen@fkf.mpg.de

Nathalie Tarrat

CEMES, Université de Toulouse, CNRS, 29 rue Jeanne Marvig, 31055 Toulouse, France E-mail: nathalie.tarrat@cemes.fr
Keywords Gold Clusters · Threshold algorithm . DFTB · Energy landscape - Probability Flows . Disconnectivity Tree

Dedicated to Dr Fernand Spiegelman on the occasion of his retirement.

\section{Introduction}

Over the past decades, complex energy landscapes that exhibit multitudes of local minima and a broad range of energy barriers have left the realm of mathematics and optimization theory, [1-4] where they are often known as cost functions, to become an indispensable playground and tool for investigations of biological, chemical and physical systems,[5] ranging from studies of structural glasses[6-10] and spin glasses, [1113] and inverse problems, over macromolecules such as proteins, [14, 15] peptides,[16-19] glycans, [20, 21] and clusters[22-24] to crystalline compounds[25, 26] and questions of evolution.[27-29] Energy landscapes are used to describe and visualize the state space of such systems, and the analysis of their barrier structure provides insight into their dynamics on short and long time scales. Regions of the landscape of chemical systems, which are locally ergodic on given observational time scales, $[30-32]$ correspond to metastable compounds or conformations, and the properties of the transition regions connecting these locally ergodic regions control both the life-times of the various modifications and the transformation pathways between them. Quite frequently, such landscapes exhibit a hierarchy of time scales, and a complicated barrier structure, making brute force simulations of their time evolution infeasible. 
A multitude of algorithmic tools, especially global optimization algorithms,[11, 33-36] have been developed to determine the local minima that are typically at the heart of the locally ergodic regions. Similarly, a variety of methods have been deployed to identify individual saddle points and pathways between given pairs of local minima. [37-40] Finally, simulations of the dynamics on complex energy landscapes have required the development of new approaches that are usually based on combinations of molecular dynamics or Monte Carlo algorithms with heuristic or systematic tools.|4146] Here, the goal is to overcome the issue of multiple time scales separated by orders of magnitude in a physically and chemically intuitive fashion, while remaining as systematical and unbiased as possible.

A major problem is the fact that fast, ie. computationally affordable, crossings of energy barriers of a certain height between two relatively close-by minima but without already prescribing a path to follow, typically require adding more energy to the system in some fashion - usually in form of temperature - than is appropriate for the phenomenon of interest. In particular, due to the complexity of the multi-minima landscape, high temperatures allow the system to range widely over the landscape moving towards many other neighbor minima instead of the "target" local minimum. Similarly, in a high-dimensional space, it becomes rather difficult to even find the exit from (or entrance to) a given minimum since the walker representing the system will spend much of the time just searching for this typically very small exit region connecting the basin to the rest of the energy landscape. This multitude of available pathways, with the resulting difficulty of the system to pick a particular one, is an example of the second class of barriers, which are prominent on a complex energy landscape: the so-called entropic barrier.[32, 47, 48] Typically, the importance of such barriers for the dynamics rapidly increases with size of the system; often they are actually more relevant than the existing lowest energy barriers regarding the time scales on which a metastable system with many degrees of freedom evolves. However, most standard exploration algorithms either ignore entropic barriers or provide no way to measure them in a systematic fashion.

One class of approaches that systematically determine such entropic barriers are the so-called lid or threshold algorithms, $[3,13,32,49-51]$ where increasingly large pockets of the energy landscape that are accessible below a sequence of energy lids or thresholds are systematically explored and the probability flows between the regions that are connected below a given lid are measured as function of threshold energy. [52, 53] Typically, one speaks of a lid algorithm, if the under- lying state space is discrete, e.g. for a spin glass[13] or a combinatorial optimization problem[3], and of the threshold algorithm if the state space is continuous, e.g. in chemical systems.[49, 50] The great advantage of measuring such probability flows as function of threshold energy is the fact that we can separate the entropic and the energetic aspects of the barrier structure of the landscape.[32, 52, 53] Furthermore, starting from e.g. a low-energy local minimum, this approach yields an overview over the neighboring minima on the energy landscape, and identifies the energetic and entropic barriers separating them. Repeating this lid based exploration from the newly discovered minima, it becomes possible to map the whole landscape in a mosaic fashion, and to reconstruct both the energy barrier and entropic barrier based connectivity of the landscape.

Since unbiased explorations of energy landscapes always require very large numbers of energy evaluations, any application of such an algorithm runs into feasibility problems for systems with expensive energy calculations such as chemical compounds. Dealing with this issue by employing e.g. empirical potentials instead of $a b$ initio energy calculations can lead to more or less grave inaccuracies, especially if the empirical potentials are fitted to only a few local minima but do not yield a faithful representation of the whole landscape. As a consequence, the minima found on the empirical landscape may be different from those on the ab initio landscape - both with regard to their existence and their relative energies.[53, 54] Furthermore, the energetic barriers between the minima will more or less strongly depend on the energy function, and similarly the entropic barriers may also differ as a consequence.

While there is no perfect solution to this conundrum, one promising compromise for chemical systems is to employ approximated $a b$ initio energies, such that we expect that essentially all local minima of the "true" $a b$ initio landscape will exist on the simplified landscape, that their relative energies are realistic, and that also the energy barriers will not differ too much from the "true" ones. One very promising approach is to employ the so-called Density Functional based Tight Binding (DFTB) method, which has proven to be quite successful in modeling many types of chemical systems and nanoclusters. [55-57] In the present work, we report the incorporation of the threshold algorithm into the DFTB code deMonNano[58].

This threshold/DFTB scheme is then used to investigate the energy landscape of gold clusters. Such clusters have attracted much interest since the 1980s because of their remarkable properties in various fields such as medicine[59], catalysis [60], nano-electronics[61] and nano-optics[62]. Among the vast literature focused 
on gold clusters [63-110], numerous works are devoted to $\mathrm{Au}_{20}[63,64,66,73,74,80,88,99,107]$ because this cluster presents a double magic number: its atomic structure is a highly symmetrical pyramid and, in the simple spherical Jellium model, its 20-electron outer electronic shell is closed $\left(1 \mathrm{~s}^{2} 1 \mathrm{p}^{6} 1 \mathrm{~d}^{10} 2 \mathrm{~s}^{2}\right.$ superorbital configuration). In recent studies, employing a new adaptation of DFTB parameters [109, 111, 112], we have investigated the potential energy surface (PES) of $\mathrm{Au}_{20}^{(0,+,-)}$ by combining a Parallel Tempering Molecular Dynamics (PTMD) exploration [113] with periodic quenching to obtain isomers and compute their structural excitation and vibrational spectra[114-116]. We have observed that the $\mathrm{Au}_{20}^{(0,+,-)}$ lowest energy isomers exhibit pyramidal shapes, highly symmetric $\left(T_{d}\right)$ for the neutral one and with very small Jahn-Teller deformations from the $T_{d}$ symmetry for the ions [114116]. These findings are in agreement with the literature, both experimental (IR[63], Trapped Ion Electron Diffraction [117], ion mobility[118] and atomic imaging [119]) and theoretical[63, 120]. Our work has shown that the structural excitation spectra of $\mathrm{Au}_{20}^{(0,+,-)}$ exhibit a difference in the first isomerization energy gap, which is larger for the neutral cluster than for the ions.

In the case of $\mathrm{Au}_{20}^{-}$, all the identified isomers with energies below $0.45 \mathrm{eV}$ above the pyramidal global minimum energy exhibit noticeable distinctive variations around the pyramidal shape, making this cluster a very suitable system to unravel the possible paths between isomers, and between groups thereof, on the PES with the help of the threshold algorithm. Beyond highlighting the connected isomer basins, we will be able to measure the probability flows between them and determine the ranges of energy where they become connected, and furthermore allow the identification of attractive basins.

In the first part of the paper, computational details are given concerning the DFTB potential used, the threshold algorithm, and the specificity of its application to the study of the $\mathrm{Au}_{20}^{-}$cluster. In the second part, the zoology of the relevant isomers is presented. Their connectivity as function of the system's energy, their proximity on the PES, and the dynamical evolution (probability flows and transition pathways) between them are discussed. Finally, a conclusion and perspectives are given.

\section{Methods}

\subsection{The DFTB potential}

In the present work, we have used the DFTB scheme $[121,122]$ in its second order formulation (also known as Self-Consistent Charge, SCC-DFTB[123]). With respect to DFT, the three main approximations of this method are that i/ the Kohn-Sham orbitals are expanded on a set of minimal atom-centered bases, ii/ the three-center integrals are neglected and iii/ the DFT energy is expanded in a Taylor series up to the second order around a reference density. In practice, the non-diagonal elements of the overlap and Hamiltonian matrices are tabulated from DFT calculations and expressed as functions of interatomic distances (the diagonal elements of the Hamiltonian matrix being the orbital energies of the isolated atoms) and the diatomic repulsive atomic pairwise contributions are fitted to minimize the difference to reference calculations.

The DFTB parameters used were adapted[112, 116, 124] from those reported by Fihey et al. [111] (the "auorg" set from the www.dftb.org website). The modification consisted in a shift of the p orbital energy [112]. In an earlier study, we have shown that these modified $\mathrm{Au}$ parameters satisfactorily reproduce the properties of systems ranging from clusters $(2 \mathrm{D} / 3 \mathrm{D}$ transition, dependency upon charge state) to bulk (lattice parameter, binding energies, elastic constants) [112]. Moreover, in the medium-size regime we are currently interested in, the structure and energetic ordering of the low-energy isomers are in agreement with the experimental and DFT data reported in the literature $[112,114,125]$. The performance of these parameters with respect to the modelling of medium-sized aggregates opens the way to the possibility of carrying out a global exploration of the PES of such aggregates with an approach providing an explicit description of the electronic structure.

For the single point energy calculations, we have used a criterion of $10^{-8}$ on the atomic charges for the SCC convergence.

\subsection{The threshold energy method}

The threshold algorithm is employed to systematically explore many low-energy pockets of the energy landscape of a given system.[32, 49, 50] A pocket is defined as the region of the state space, which is accessible from a given starting point, usually a local minimum, below a given energy lid or threshold.[126] The exploration of the pocket typically takes place via many Monte Carlo random walks starting from the same minimum, but can also be performed using molecular dynamics or combinations thereof. $[32,51]$ This ensemble of random walkers starting from the same point represents a unit of probability that has been placed into the starting minimum. Keeping track of this ensemble of walkers then corresponds to measuring the flow of probability on the energy landscape as function of threshold energy. 
A crucial element is that each random step from point $i$ to point $i+1$ is always accepted as long as the energy of state $i+1$ remains below the given energy lid, while any step above the lid is always rejected.

Periodically, every $\mathrm{N}_{\text {stop }}$ random moves, we perform a set of many stochastic quenches, i.e. random walks where only states $i+1$ with lower or equal energy than the current state i are accepted. Each stochastic quench is followed by a local optimization. From these sets of optimized structures, we can deduce the probability flows in the pocket between the starting minimum and the other minima inside the pocket as function of energy lid and length of threshold run. One should note that due to the rapid increase in the number of states with energy on complex energy landscapes, a large fraction of the stopping points will be close to the energy threshold. Thus, the probability flows directly reflect the entropic barriers inside the pocket for a given energy interval close to the lid. In practice, we define the probability flow $p_{i \rightarrow j}$ from isomer $i$ to isomer $j$ as follows: For all the $N_{i}^{\text {tot }}$ threshold simulations starting from an isomer $i$, we define $N_{i}^{j}$ as the number of final structures (after conjugate gradient optimization) corresponding to a basin $j \cdot p_{i \rightarrow j}$ is then defined as $N_{i}^{j} / N_{i}^{t o t}$.

This procedure is repeated for a sequence of energy lids for the same starting minimum, and subsequently for (ideally all) other local minima on the energy landscape, where not only the original set of starting minima - which had been obtained from e.g. a global optimization - but also the local minima discovered during the threshold runs are included. Thus, we obtain local densities of states as function of lid and starting local minima, and probability flows as function of energy lid and starting minima.

Furthermore, we can employ the probability flows to deduce a tree graph representation of the energy landscape, where we connect any two minima at the energy, for which there had first been an uninterrupted path feasible between these minima regardless of how far away they are on the landscape. $[3,50,52,53] \mathrm{We}$ note that this path may well consist of many individual pieces, each connecting two close-by local minima, since for large energy landscapes the number of steps in a given threshold run will often be very small compared to the total accessible state space volume below the lid, and thus two minima that are very far from each other but still connected below the lid are unlikely to be reached during a single individual threshold run.

\subsection{Application to $\mathrm{Au}_{20}^{-}$}

For the moveclass during the threshold runs, we selected individual atom displacements. We have inves- tigated five different lid energies (Lid1-5), corresponding to $0.01 / 0.02 / 0.03 / 0.04 / 0.05$ Hartree above the energy of the global minimum configuration. For each minimum chosen as starting point and below each energy lid, at least 30 different threshold runs, i.e. random walks below the lid energy, were performed. Along each threshold run we stopped 75 times, and performed 4 quenches at each stopping point. Each piece of the threshold run between two stopping points consisted of $5000 \mathrm{MC}$ steps, and each of the 4 quenches consisted of 500 MC steps followed by a conjugated gradient optimization. We have used a criterion of $4 \cdot 10^{-6}$ a.u. for the maximum atomic force to stop the local optimization.

In order to classify and compare the structures, we have used as a first criterion the energy differences. Two structures are considered as similar if their energy difference is below $10^{-9}$ Hartree. In addition, we employed a structural similarity criterion, namely the interatomic distance-based similarity function introduced by Joswig et al.[127]. Two structures are assumed to be similar if this criterion is higher than 0.98. Note that, in our approach, mirror symmetric images are considered to belong to the same basin.

\section{Results}

\subsection{Isomer analysis}

The simulation protocol was initiated using as starting structures the 10 isomers reported as the lowest energy ones in the references $[115,116]$. Additional isomers identified during an iteration of the DFTB/threshold scheme were added to the list of starting structures if they met one of the two following criteria: i/ a total energy differing from the one of the pyramid, assumed to be the global minimum, by less than $0.45 \mathrm{eV}$ or ii/ that a given minimum $j$ represents more than $10 \%$ of the optimized structures issued from a given starting configuration $i$ at a given lid $\left(p_{i \rightarrow j}>10 \%\right)$. This procedure has been iterated until there was no longer a new structure that meets these criteria. The total number of minima that have been identified is 736 . These isomers are labelled by a number $y$ according to the increasing energetic order. Among them, 40 isomers have been used as starting structures (45 structures met the criteria given above, but 5 were removed because they corresponded to very small structural deformations of isomers already included in the list of starting structures). These 40 structures can therefore be considered as corresponding to key basins on the potential energy surface because of their low energy and/or their high probability of being visited. In the following, these 40 starting structures will be referred as "key isomers". 


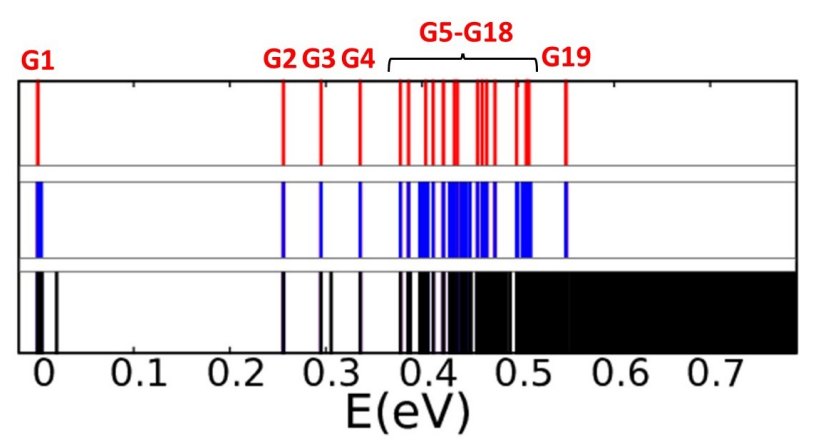

Fig. 1 Structural excitation energies of isomers (eV). Black: the full set of 736 optimized isomers. Blue: the 40 key isomers (used as starting points for structural explorations, see text). Red: lowest energy isomers of the 19 identified structural groups and labels identifying the groups.

The structural excitation energy of these 40 key isomers and of all the minima, with respect to the pyramid, are represented in figure 1 (blue and black spectra, respectively). This structural excitation spectrum is discrete up to about $0.4 \mathrm{eV}$, at which point it begins to take on a continuous aspect. The first four isomers $(y=1,2,3,4)$ are slightly distorted pyramids, the isomerization gap between these pyramid-like structures and the higher energy isomers being about $0.24 \mathrm{eV}$, which is consistent with our previous work [116].

The 40 key isomers have been classified into 19 structural groups by eye. The structure of the lowest energy isomer of each group is shown in figure 2 , and the corresponding structural excitation energies appear in figure 1 (red spectrum). These structures will be denoted $\mathrm{G} x-y, x$ being the identifier of the structural group, $y$ being the isomer number. The first two groups, G1 and G2, correspond to pyramid-like structures, G1 containing very slightly deformed pyramids, G2 comprising a pyramid-like structure but with one of its facets being convex. At higher energies, there are three groups containing overall pyramid-like shapes, but with noticeable distortions, either on part of the structure (G6) or on the whole (G9 and G16). In seven groups, the structures can be described as a pyramid where the atom of one of the facets has been removed, leaving a hole, and placed either on the same facet (G3), or at different positions on another facet (G5, G13 and G18), or on an edge (G10 and G15). The seventh group of this type is G19 which, although very distorted, also exhibits this type of structure. The other structural groups are based on a pyramid on which an atom located at a vertex has been removed and placed either on an edge (G4, G12, G14) or on a facet (G7, G8, G11, G17). Interestingly, the 19 structural groups described above are reminiscent of the structural classification re- ported for low-lying isomers of neutral $\mathrm{Au}_{20}$ by Aprà et al. [107], namely Td followed by either defective Td or compact forms.

\subsection{Disconnectivity analysis}

A tree graph is presented in figure 3 (tree graphs are also sometimes called disconnectivity graphs [128], disconnectivity diagrams[129] or one-dimensional projections [130]). On this graph, the energy used to place each group corresponds to that of its lowest energy isomer, the blue dashed lines show the threshold energies at which the explorations were carried out (Lid1 to Lid5), and the red lines show which groups are connected at a given lid energy. Note that these lids correspond to upper limits of the energies required to connect the groups.

Below Lid1, only the first two groups (G1 and G2) exist and there is a path connecting them. For all other groups, the energy of their lowest energy isomer is located between Lid1 and Lid2, except for group G19. In this case it is almost equal to Lid2. Between Lid1 and Lid2, there are paths to connect groups G1, G2, G4, G6 and G9. All these groups present variations around the pyramid, either a vertex atom that shifts slightly to bridge a single edge, or global deformations. Groups G5 and G18, which are very close structurally, are also connected below Lid2.

At Lid3, the set containing G1, G2, G4, G6 and G9 is extended to include G7 and G16. This union of groups seems intuitive because of the compact pyramidal base of the latter (note that, at this lid, G16 is divided into two distinct subgroups, both of them linked to the set containing G1). Moreover, group G15 becomes accessible from the set containing G5+G18, these three groups having a common pattern, namely a pyramid with a hole on one facet. In addition, three new sets of groups appear at Lid3. The basins G10 and G13 are connected, the latter exhibiting a hole and differing only by the position of the adatom at the center of a triangle of a facet or on the edge of such a triangle. In a similar way, basins G8 and G12 are connected, the difference being that the adatom in this case does not come from a hole created in the middle of a facet but from a vertex. The last set that appears at Lid3 contains the groups G11, G14 and G17. They are also based on a pyramid from which one vertex has been removed but, unlike the G8 group, the adatom is not linked to the facet opposite to the removed vertex but on an adjacent one. All the groups that are connected at Lid3 exhibit strong structural similarities, such that both the energy barriers and the entropic barriers are small enough to be crossed at this lid. 


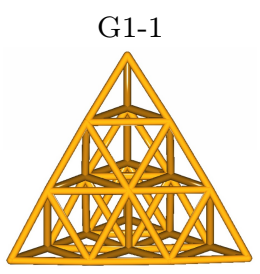

G6-10

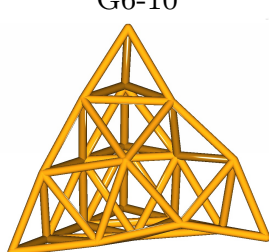

G11-23

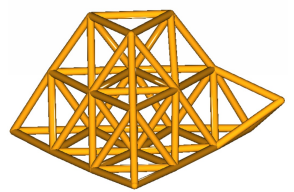

G16-75

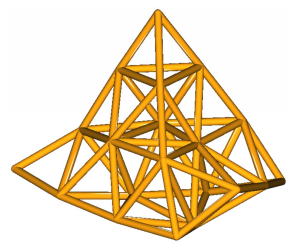

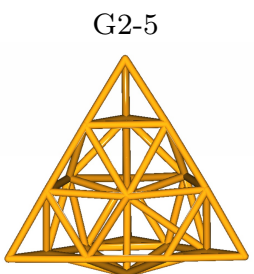

G7-15

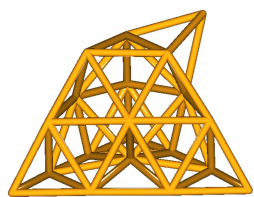

G12-33

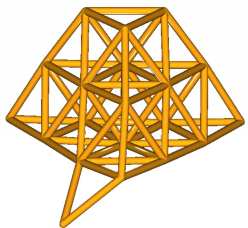

G17-94

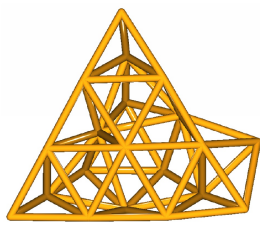

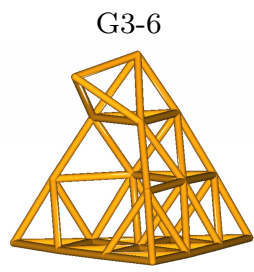

G8-17

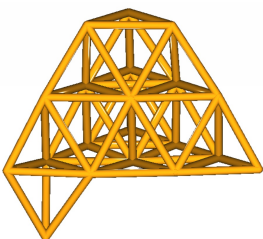

G13-39

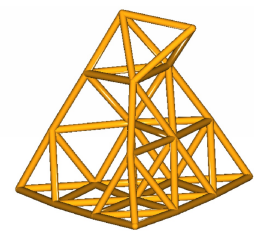

G18-96

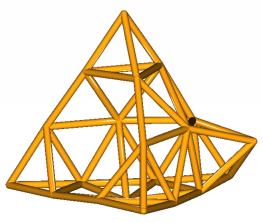

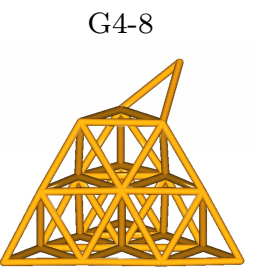

G9-18

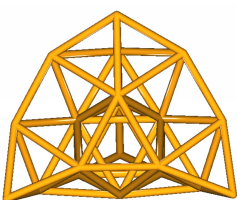

G14-45

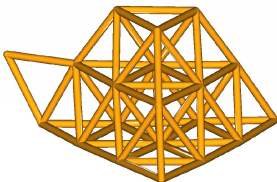

G19-152

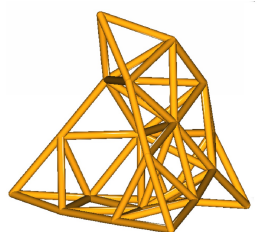

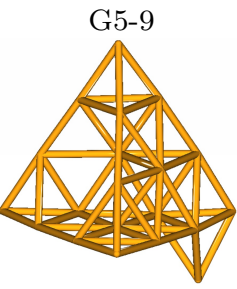

G10-22

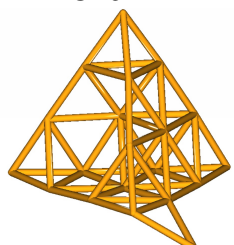

G15-54

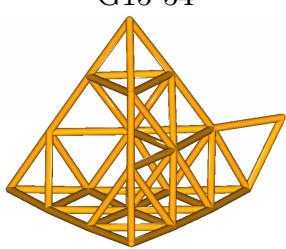

Fig. 2 Lowest energy isomers of the 19 groups.

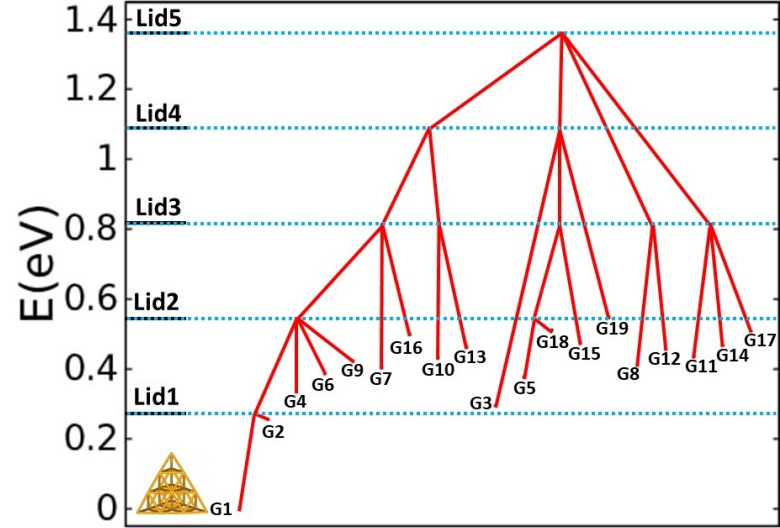

Fig. 3 Disconnectivity graph showing the relative total energies (in $\mathrm{eV}$ ) of the lowest energy isomers of the 19 groups with respect to the energy of the pyramid. The blue dashed lines indicate the energy lid positions.

At Lid4, there are only two changes. The set containing groups G10 and G13 is now linked to the set containing G1. This is the first connection between isomers based on a pyramid exhibiting either a missing vertex or a hole on one of its facets. The second change occurring at Lid4 is that the set containing
G5 + G18+G15 now also includes groups G3 and G19. At Lid5, there are paths on the potential energy surface that connect all the 19 groups.

\subsection{Proximity and probability flows}

In this section, we attempt to provide a picture of the basins' proximity on the PES as well as an analysis of the dominant probability flows, on the basis of the analysis of the threshold simulation performed at the highest lid, i.e. at 0.05 Hartree above the pyramid global minimum. This lid has been chosen as it is the first one to connect all the identified basins. In the supplementary materials, two figures S1 and S2 show all the probability flows $p_{i \rightarrow j}$ between the 40 key isomers. These pictures show that some routes are favored with respect to others, motivating an extraction of dominant fluxes, which are depicted on figure 4 . The top panel allows to visualize the main fluxes between isomer basins belonging to a given group $\mathrm{G} x$ (called intragroup fluxes) whereas the bottom panel shows the main fluxes between groups (called intergroup fluxes).

In figure 4-top, an arrow represents an intragroup flux from a key isomers $i$ to a key isomer $j$ if $p_{i \rightarrow j}$ is 


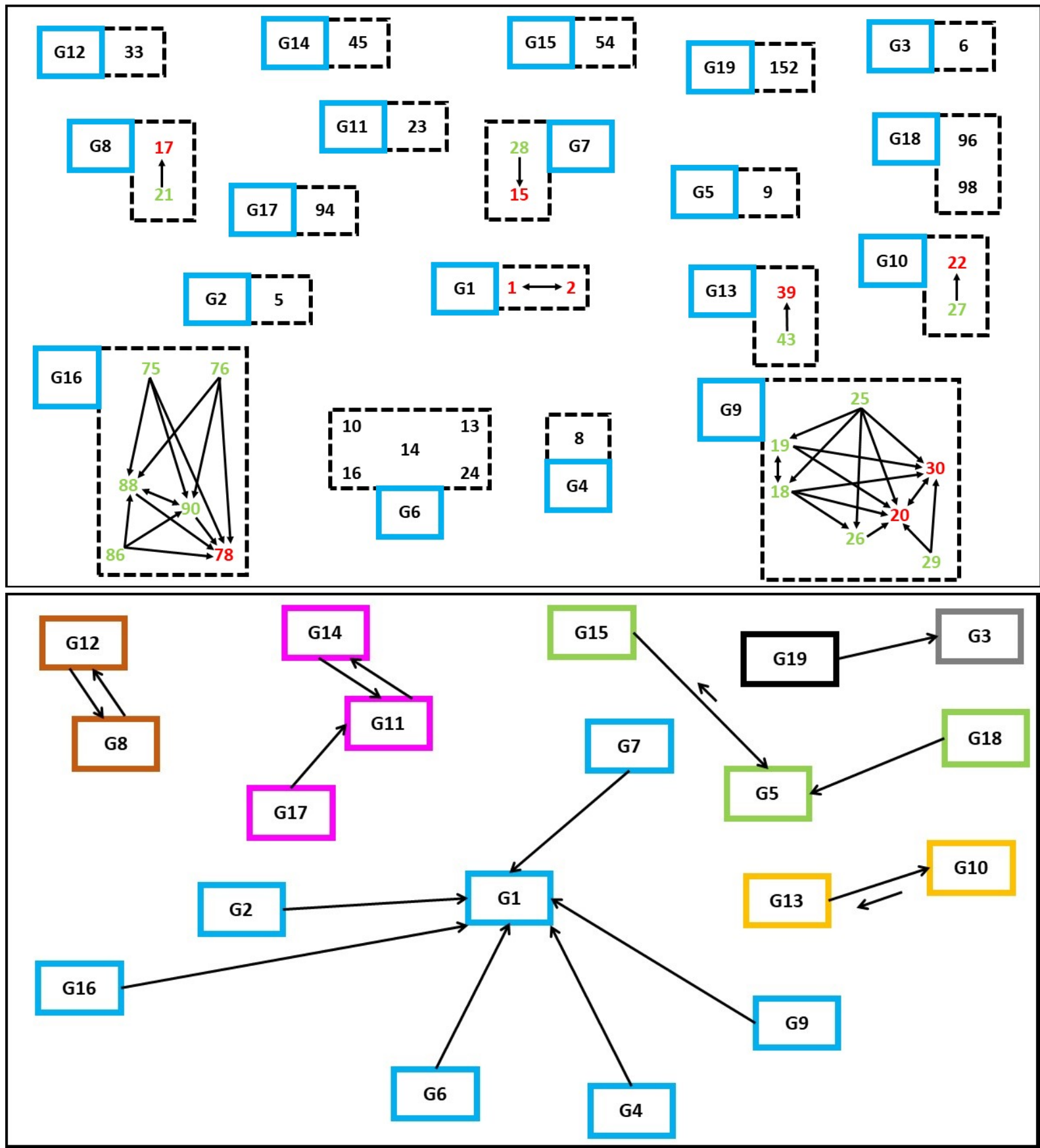

Fig. 4 Fluxes at Lid5. Top : Fluxes between key isomers within the 19 groups. The identifiers of the isomers are in green except if there is no significant flux within a given group (isomers in black), or if they correspond to an attractive basin (isomers in red). Bottom : Fluxes between the different groups. The colours of the boxes in the bottom figure identify the members of the 7 super-groups at Lid3.

larger than $5 \%$. If a significant flux is present in both directions between two key isomers $\left(p_{i \rightarrow j}\right.$ and $p_{j \rightarrow i}$ both larger than $5 \%$ ), then only the dominant flux is represented, except for the cases where the largest one is less than twice the smallest one. In the latter case, a double arrow is plotted. When two or more key isomers belong to the same group, the isomer identified as an attractor is depicted in red whereas a green color is used for the others. Starting with G1, an equilibrium is observed between the various pyramidal forms, particularly in the case of isomers 1 and 2, selected as starting points. In the groups G7, G8, G10 and G13, each one containing 2 key isomers, the lowest energy isomer (the one with the smallest index number) always appears as the at- 
tractive region of the group. A more complex picture is found for groups G16 and G9, which contain several key isomers. In group G16, the two lowest energy key isomers, namely 75 and 76, appear to correspond to non-attractive basins. This is also the case for isomer 86. Indeed, one-way-fluxes are observed from these isomers towards the three other ones (88, 90 and 78). Among them, the basin of the key isomer 78 appears to be the most attractive one. In group G9, the two lowest energy key isomers (18 and 19) do not correspond to attractive basins either. In this group, the attractive region corresponds to a pair of basins, namely those of isomers 20 and 30. These isomers seem to benefit from their intermediate position between two different parts of the PES, namely the one corresponding to isomer 29 and the one corresponding to isomers 18, 19, 25 and 26. As remarked above, in groups G9 and G16, the most attractive key isomer does not correspond to the one with the lowest energy. Actually, at a given lid energy, the relative fluxes between basins depends on their relative accessible phase space volume and this volume may or may not correlate with the depth of the minimum. Finally, groups G6 and G18 present a different picture as no intragroup flux could be identified. This is simply because each key isomer of these groups undergoes a very efficient transformation toward a more symmetric shape, joining, respectively, groups G1 (for G6 isomers) and G5 (for G18 isomers) before visiting other isomers of their own group. Obviously, the number of fluxes depicted in this figure depends on the $p_{i \rightarrow j}$ limit value chosen for extracting the dominant fluxes. The choice of a limit value of $5 \%$ was motivated by the intention to highlight the most likely paths only. Minority flows not taken into account in this analysis, i.e. with $p_{i \rightarrow j}<$ $5 \%$, can be observed in figure S2 of the supplementary information. From this figure, we can see for instance that with a $p_{i \rightarrow j}$ limit value of $1 \%$, all the isomers of G16 are connected.

We now address the intergroup fluxes. We first define for each key isomer $i$ its outside group flux toward the group GJ as : $p_{i \rightarrow G J}=\sum_{j \in G J} p_{i \rightarrow j}$. We observed that the outside group fluxes are similar for all key isomers of a given group $G I$, allowing to define intergroup group fluxes; i.e. $\forall i, i^{\prime} \in G I, p_{i \rightarrow G J} \simeq p_{i^{\prime} \rightarrow G J} \simeq$ $P_{G I \rightarrow G J} \cdot{ }^{1}$ For each group $G I$, the main outside group flux (the largest value for $P_{G I \rightarrow G J} \forall G J$ ) is represented by an arrow in figure 4-bottom. When two groups are connected in both directions, the relative length of the two arrows is proportional to the relative intensity of these fluxes. The groups connected by arrows on fig-

\footnotetext{
1 Note that this also validates the use of the groupings as physically meaningful entities, possibly corresponding to large locally ergodic regions.
}

ure 4-bottom define a super-group. The first supergroup contains the pyramid group G1, which appears to be very attractive, as no simulation starting from this group visited another one, plus 6 other groups G2, G4, G6, G7, G9 and G16, all of them attracted by G1. In the other super-groups, probability flows occurring in two directions could be identified for four pairs of groups, namely G8 and G12; G11 and G14; G5 and G15; G10 and G13. In all cases, these fluxes correspond to small displacements of an adatom moving from the top of a triangle toward an edge. Although the present discussion is done for the highest lid $(0.05 \mathrm{Ha})$ it is interesting to compare the connections made on the basis of these probability flows with those identified from the disconnectivity graph (figure 3). In figure 4-bottom, the colors represent the groups that are connected at Lid3. It appears that the flux based picture obtained here at Lid5 almost perfectly matches the connectivity map at Lid3. Indeed, the groups which are connected at Lid3 belong to the same super-group at Lid5, with the single exception of groups G19 and G3, which are not connected at Lid3. One should mention, however, that these two groups are connected at Lid4, together with groups G5, G15 and G18. This comparison of the disconnectivity graph and the main intergroup fluxes at Lid5 provides a consistent representation of the basins' proximity.

The G1 basin is the most attractive one and therefore the most likely to be observed; this nicely concurs with already reported results, such as the agreement between the experimental TIED spectrum and the slightly distorted Td isomer theoretical spectrum for $\mathrm{Au}_{20}^{-}[65,66]$. Those authors concluded that the other investigated motifs such as icosahedron and decahedron can be clearly excluded as major contributors. This result was interpreted in terms of the energetic stability of the pyramid isomer with respect to the few other guessed structures. This is in line with our current work involving an unbiased exploration of the low-energy region of the landscape encompassing all relevant isomers and a study of the probablity flows taking energetic and entropic barriers into account. Note that the attractivity of the pyramid basin has also been reported from DFT-basin hopping explorations in the case of neutral $\mathrm{Au}_{20}$ [107].

\subsection{Dynamical evolution}

The analysis of the probability flows allows for a detailed understanding of the structural deformations that occur when connecting different groups. When the transformation is not obvious, an additional analysis of the stopping points visited during the trajectory connecting two groups provides a complementary insight. 
In our analysis, the intuitive migration of an adatom over the cluster surface never appeared to be an efficient way to connect two isomers. We have identified three main characteristic moves which are described in the paragraph below.

The first type of transformation is a global deformation of the cluster involving all atoms. In this category, one can find transitions between the pyramid G1 and the groups corresponding to a deformed pyramid, namely G2, G6, G9, G16, occurring at low energy (Lid2). The global deformation is also a way to connect two isomers through a third one that exhibits a mixture of their characteristic features. This is, for instance, the case in the transition from key isomer G3-6 (adatom close to the surface-hole) toward key isomer G5-9 (adatom far from the surface hole) which are connected at Lid5 through the key isomer G19-152. This isomer presents a surface hole and two atoms deviating from their position in the corresponding pyramid (one close and one far from the hole). A collective motion allows the one close to (respectively far from) the hole to recover its place as a vertex, the other one becoming the adatom, leading to key isomer G5-9 (respectively G36 ). The second transformation type is the displacement of an adatom close to a vertex from the top of a triangle toward the top of an edge. Such a transformation connects groups G8 and G12; G11 and G14; G5 and G15; G10 and G13 at Lid3 and above. The third type of transformation involves the migration of an atom to fill a hole in a facet center. Two illustrations of such trajectories are shown in figure 5, connecting the group G13 to groups G5 and G1, respectively. In the trajectory connecting the key isomers G13-39 and G5-9, the adatom (green) is almost not perturbed and the transformation proceeds via the migration of a facet atom (pink) through the center of the pyramid toward the hole, creating a new hole in its original position. In the second trajectory, G13-39 $\rightarrow$ G1-1, the recombination proceeds in a concerted mechanisms, an atom (pink) from an edge located between the hole and the adatom (green) moves toward the hole while its original place is taken by the adatom.

On the basis of this analysis, it is interesting to point out that the structural proximity of some isomers may lead to the design of intuitive paths that are actually not the most likely ones. As an example, it is intuitive to think that the key isomer G3-6 is connected to the pyramid group G1 through a simple motion of the adatom toward the hole. However, in our simulations G3 and G1 have only been connected at Lid5 through a more complex path involving two global cluster deformations (from G3-6 to G19-152 first and from G19-152 to G59) and one hole migration (G5-9 to G13-39, top line of figure 5) followed by the hole filling via the concerted mechanism from G13-39 to G1-1 (bottom of figure 5). In the same way, the key isomer G11-23 is not connected to the pyramid by a migration of the adatom on the surface to reach the depleted vertex. This isomer is actually connected to G1 via the migration of the adatom on top of a triangle toward an edge bridging position (G11-23 to G12-33) followed by global deformations $(\mathrm{G} 12-33 \rightarrow \mathrm{G} 16-75 \rightarrow \mathrm{G} 1-1)$.

A similarly counter-intuitive pathway has been reported by Schebarchov et al. [108] for a larger neutral gold cluster, namely $\mathrm{Au}_{55}$. They observed that the intuitive rearrangement via adatom hops is not the fastest route to connect two close-packed isomers; instead, a concerted mechanism is more efficient. Interestingly, our conclusion regarding the paths where an adatom is filling a hole on a planar facet of an $\mathrm{Au}_{20}^{-}$cluster is quite analogous to their observation of a concerted move sequence between two fcc-minima in $\mathrm{Au}_{55}$. We further note that rearrangement paths involving collective moves also appear to be dominant paths in small nanoalloys [131-133].

\section{Conclusions}

The threshold algorithm is a versatile tool to explore complex potential energy surfaces and, in particular, their barrier structure. It provides an overview of the groups of neighboring minima existing on the energy landscape and yields upper limits of the lowest energy barriers between them. In addition, it allows the identification of transition pathways connecting minima basins and individual minima and the measurement of the probability flows between them, thus yielding information about both energetic and entropic barriers on the landscape. Since unbiased explorations of energy landscapes require huge numbers of energy calculations, and such calculations are very expensive computationally for chemical systems on the ab initio level, we have incorporated the threshold algorithm in the DFTB deMonNano code [58]. Indeed, the computational efficiency of DFTB with respect to DFT makes it a tool of choice to investigate the energy landscape of intermediate and large systems, for which a quantum description is mandatory. This new DFTB/threshold scheme has been applied to the study of an anionic metal cluster : $\mathrm{Au}_{20}^{-}$. This system exhibits a complex potential energy surface containing many isomers and a wide range of energy barriers, and requires a treatment at the quantum level to ensure that the landscape exhibits all relevant isomers in the correct energetic order and that the energetic and entropic barriers separating them are reliable. 


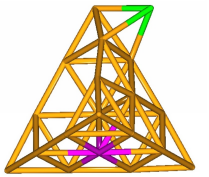

G13-39

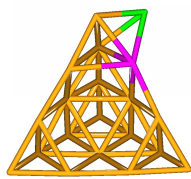

G13-39
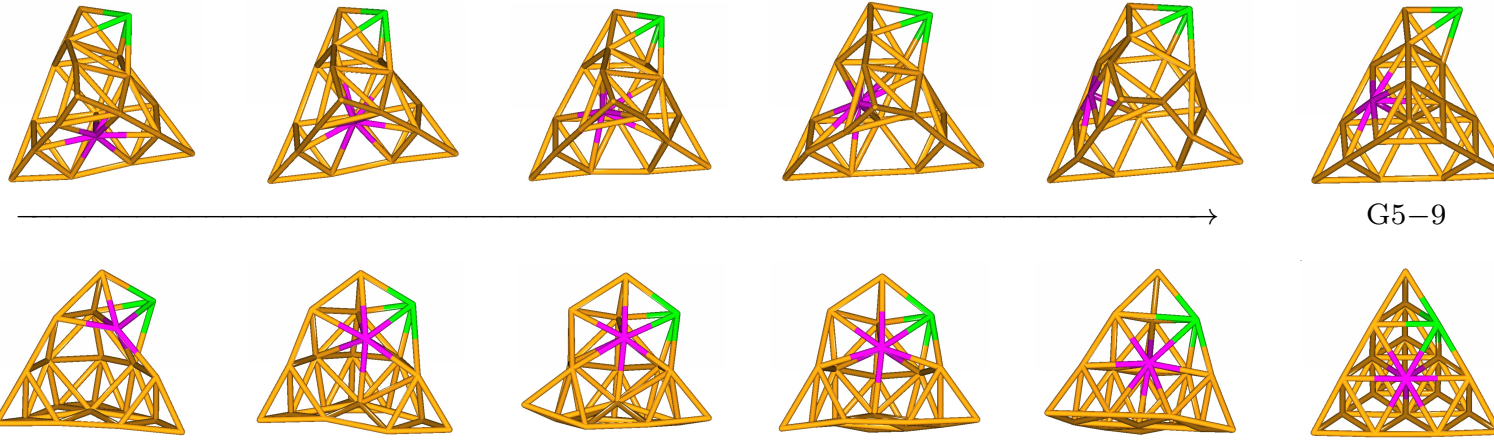

G1-1

Fig. 5 Illustration of transition pathways. Top: trajectory connecting the key isomers G13-39 and G5-9; the adatom (green) is almost not perturbed and the transformation proceeds via the migration of a facet atom (pink) through the center of the pyramid toward the hole, creating a new hole in its original position. Bottom: trajectory connecting the key isomers G13-39 and G1-1; the recombination proceeds in a concerted mechanisms, an atom (pink) from an edge located between the hole and the adatom (green) moves toward the hole while its original place is taken by the adatom. The cartesian coordinates corresponding to these pathways are given in Supplementary Material.

The exploration allowed to identify 736 local minima on the PES. Among them, 40 key isomers have been identified using energetic and occurrence probability criteria. On the basis of geometrical considerations, these 40 key isomers could be distributed in 19 groups. Each group can be seen as corresponding to structures with a pyramidal shape with three specific variations : i/ distorted pyramids, ii/ pyramids in which the atom of one of the facets has been removed, leaving a hole, and placed either on the same facet, at different positions on another facet, or on an edge, and iii/ pyramids on which an atom located at a vertex has been removed and placed on an edge or on a facet. Upper limits of the energy barriers separating the different groups on the PES were evaluated, and at the highest investigated energy threshold $(0.05 \mathrm{Ha})$, all groups were found to be connected. Furthermore, the probability flows between basins of the PES were analysed at this lid value, both inside each group and between different groups. This allowed us to define attractive isomers within groups and attractive groups. Interestingly, in a given group, the key isomer with the lowest energy was not always found to be the most attractive one. The analysis of the fluxes provided a representation of the $\mathrm{Au}_{20}^{-}$basins' proximity in good agreement with the disconnectivity tree. Finally, detailed information about transition pathways between the various isomers has been obtained from the analysis of the threshold runs, leading to the identification of counter-intuitive transformation mechanisms. In particular, it appeared that the migration of an adatom over the $\mathrm{Au}_{20}^{-}$cluster surface is not an efficient way to connect two isomers and that more complex concerted mechanisms are involved.

In summary, the suitability of the DFTB/threshold scheme developed in the present work has been clearly demonstrated in the context of the complex $\mathrm{Au}_{20}^{-} \mathrm{PES}$ analysis. We believe that this work should pave the way toward applications to other systems, ranging from molecules to nanoparticles, both in vacuum and on surfaces.

\section{Acknowledgements}

This work was granted access to the HPC resources of the CALMIP supercomputing center (Grant p18009).

\section{Supplemental Material}

Cartesian coordinates of the lowest energy isomers of the 19 groups. Cartesian coordinates corresponding to the illustration of transition pathways depicted in figure 5 (from group G13 to group G5 and from group G13 to group G1). Probability flows between the 40 key isomers at Lid5.

\section{References}

1. V. Czerny, J. Optim. Theo. Appl. 45, 41 (1985)

2. S. Kirkpatrick, G. Toulouse, J. Phys. 46, 1277 (1985)

3. P. Sibani, J.C. Schön, P. Salamon, J.O. Andersson, Europhys. Lett. 22, 479 (1993)

4. P. Salamon, P. Sibani, R. Frost, Facts, Conjectures, and Improvements for Simulated Annealing (SIAM Monographs, Philadelphia, 2002) 
5. D.J. Wales, Energy Landscapes with Applications to Clusters, Biomolecules and Glasses (Cambridge Univ. Press, 2004)

6. F. Stillinger, Science 267, 1935 (1995)

7. J.C. Schön, P. Sibani, J. Phys. A: Math. Gen. 31, 8165 (1998)

8. K.K. Battacharya, K. Broderix, R. Kree, A. Zippelius, Europhys. Lett. 47(4), 449 (1999)

9. J.C. Schön, P. Sibani, Europhys. Lett. 49, 196 (2000)

10. U. Buchenau, J. Phys.: Cond. Matter 15, S955 (2003)

11. S. Kirkpatrick, C.D. Gelatt Jr., M.P. Vecchi, Science 220, 671 (1983)

12. P. Sibani, K.H. Hoffmann, Phys. Rev. Lett. 63(26), 2853 (1989)

13. P. Sibani, P. Schriver, Phys. Rev. B 49, 6667 (1994)

14. J. Skolnick, A. Kolinski, C.L. Brooks III, A. Godzik, A. Rey, Curr. Biol. 3, 414 (1993)

15. J.M. Troyer, F.E. Cohen, Proteins: Struct. Funct. Gen. 23, 97 (1995)

16. C. Angell, Science 267, 1924 (1995)

17. C.M. Roland, P.G. Santangelo, K.L. Ngai, J. Chem. Phys. 111, 5593 (1999)

18. J.C. Schön, J. Phys. Chem. A 106, 10886 (2002)

19. M.T. Wolfinger, S. Will, I.L. Hofacker, R. Backofen, P.F. Stadler, Europhys. Lett. 74, 726 (2006)

20. S. Abb, N. Tarrat, J. Cortes, B. Andriyevsky, L. Harnau, J.C. Schön, S. Rauschenbach, K. Kern, Angew. Chem. 131, 8424 (2019)

21. S. Abb, N. Tarrat, J. Cortes, B. Andriyevsky, L. Harnau, J.C. Schön, S. Rauschenbach, K. Kern, RSC Advances 9, 35813 (2019)

22. D.J. Wales, J.P.K. Doye, M.A. Miller, P.N. Mortenson, T.R. Walsh, in Advances in Chemical Physics, volume 115, ed. by I. Prigogine, S.A. Rice (Wiley, New York, 2000), pp. 1-111

23. R. Ferrando, J. Jellinek, R.L. Johnston, Chem. Rev. 108, 845 (2008)

24. R. Pacheco-Contreras, M. Dessens-Felix, D.J. Borbon-Gonzalez, L.O. Paz-Borbon, R.L. Johnston, J.C. Schön, A. Posada-Amarillas, J. Phys. Chem. A 116, 5235 (2012)

25. J.C. Schön, M. Jansen, Angew. Chem. Int. Ed. Eng. 35, 1286 (1996)

26. S.M. Woodley, P.D. Battle, J.D. Gale, C.R.A. Catlow, Phys. Chem. Chem. Phys. 1, 2535 (1999)

27. S. Govindarajan, R.A. Goldstein, Proteins: Struct. Funct. Gen. 29, 461 (1997)

28. P. Sibani, M. Brandt, P. Alstrom, Int. J. Mod. Phys. B 12, 361 (1998)

29. J.M. Kleinberg, J. Comp. Biol. 6, 387 (1999)
30. J.C. Schön, in Proceedings of RIGI-workshop 1998, ed. by J. Schreuer (ETH Zürich, Zürich, 1998), pp. $75-93$

31. J.C. Schön, M. Jansen, Z. Krist. 216, 307 (2001)

32. S. Neelamraju, C. Oligschleger, J.C. Schön, J. Chem. Phys. 147, 152713 (2017)

33. J.H. Holland, Adaptation in Natural and Artificial Systems (Univ. Mich. Press, Ann Arbor, 1975)

34. D.M. Deaven, K.M. Ho, Phys. Rev. Lett. 75, 288 (1995)

35. D.J. Wales, J.P.K. Doye, J. Phys. Chem. A 101, 5111 (1997)

36. A. Möbius, A. Neklioudov, A. Diaz-Sanchez, K.H. Hoffmann, A. Fachat, M. Schreiber, Phys. Rev. Lett. 79, 4297 (1997)

37. A. Banerjee, N. Adams, J. Simmons, R. Shepard, J. Phys. Chem. 89, 52 (1985)

38. R.S. Berry, H.L. Davis, T.L. Beck, Chem. Phys. Lett. 147, 13 (1988)

39. R.S. Berry, Chem. Rev. 93, 2379 (1993)

40. D.J. Wales, J. Chem. Soc. Farad. Trans. 89, 1305 (1993)

41. T. Huber, A. Torda, W.F. van Gunsteren, J. Comput. Aided Mol. Des. 8, 695 (1994)

42. H. Grubmüller, Phys. Rev. E 52, 2893 (1995)

43. G.T. Barkema, N. Mousseau, Phys. Rev. Lett. 77, 4358 (1996)

44. P.G. Bolhuis, C. Dellago, D. Chandler, Faraday Discuss. 110, 421 (1998)

45. G. Wei, N. Mousseau, P. Derreumaux, J. Chem. Phys. 117, 11379 (2002)

46. A. Laio, M. Parrinello, Proc. Nat. Acad. Sci. 99, $12562(2002)$

47. J.C. Schön, M.A.C. Wevers, M. Jansen, J. Phys.: Cond. Matter 15, 5479 (2003)

48. A. Fischer, K.H. Hoffmann, J.C. Schön, J. Phys. A: Math. Theor. 44, 075101 (2011)

49. J.C. Schön, Ber. Bunsenges. 100, 1388 (1996)

50. J.C. Schön, H. Putz, M. Jansen, J. Phys.: Cond. Matt. 8, 143 (1996)

51. S. Neelamraju, R. Johnston, J.C. Schön, J. Chem. Theo. Comp. 12, 2471 (2016)

52. M.A.C. Wevers, J.C. Schön, M. Jansen, J. Phys.: Cond. Matt. 11, 6487 (1999)

53. S. Neelamraju, J.C. Schön, K. Doll, M. Jansen, Phys. Chem. Chem. Phys. 14, 1223 (2012)

54. C. Heard, J.C. Schön, R.L. Johnston, Chem. Phys. Chem. 16, 1461 (2015)

55. F. Spiegelman, N. Tarrat, J. Cuny, L. Dontot, E. Posenitskiy, C. Martí, A. Simon, M. Rapacioli, Adv. Phys.: X 5(1), 1710252 (2020)

56. M. Gruden, L. Andjeklović, A.K. Jissy, S. Stepanović, M. Zlatar, Q. Cui, M. Elst- 
ner, 38(25), 2171 (2017)

57. B.R.L. Galvão, L.P. Viegas, D.R. Salahub, M.P. Lourenço, J. Mol. Model. 26(11), 303 (2020)

58. T. Heine, M. Rapacioli, S. Patchkovskii, J. Cuny, J. Frenzel, A. Koster, P. Calaminici, H.A. Duarte, S. Escalante, R. Flores-Moreno, A. Goursot, J. Reveles, D. Salahub, A. Vela, (2015), deMonNano, http://demon-nano.ups-tlse.fr/, 1st Sept 2016

59. L. Dykman, N. Khlebtsov, Chem. Soc. Rev. 41, 2256 (2012)

60. G.J. Hutchings, J.K. Edwards, in Metal Nanoparticles and Nanoalloys, Frontiers of Nanoscience, vol. 3, ed. by R.L. Johnston, J. Wilcoxon (Elsevier, 2012), pp. $249-293$

61. M. Homberger, U. Simon, Phil. Trans. R. Soc. A 368(1915), 1405 (2010)

62. N.J. R.G., L. Frederic, Nanophotonics 6(1), 71 (2017)

63. P. Gruene, D.M. Rayner, B. Redlich, A.F.G. van der Meer, J.T. Lyon, G. Meijer, A. Fielicke, Science 321(5889), 674 (2008)

64. H. Häkkinen, Chem. Soc. Rev. 37, 1847 (2008)

65. D. Schooss, P. Weis, O. Hampe, M.M. Kappes, Philosophical Transactions of the Royal Society A: Mathematical, Physical and Engineering Sciences 368(1915), 1211 (2010)

66. A. Lechtken, C. Neiss, J. Stairs, D. Schooss, The Journal of Chemical Physics 129(15), 154304 (2008)

67. B. Yoon, P. Koskinen, B. Huber, O. Kostko, B. von Issendorff, H. Häkkinen, M. Moseler, U. Landman, ChemPhysChem 8(1), 157 (2007)

68. X.B. Li, H.Y. Wang, X.D. Yang, Z.H. Zhu, Y.J. Tang, J. Chem. Phys. 126(8), 084505 (2007)

69. A.V. Walker, J. Chem. Phys. 122(9), 094310 (2005)

70. H. Häkkinen, U. Landman, Phys. Rev. B 62, 2287 (2000)

71. H. Häkkinen, M. Moseler, U. Landman, Phys. Rev. Lett. 89, 033401 (2002)

72. H. Häkkinen, B. Yoon, U. Landman, X. Li, H.J. Zhai, L.S. Wang, J. Phys. Chem. A 107(32), 6168 (2003)

73. L. Xiao, L. Wang, Chem. Phys. Lett. 392, 452 (2004)

74. L. Xiao, B. Tollberg, X. Hu, L. Wang, J. Chem. Phys. 124(11), 114309 (2006)

75. M.P. Johansson, A. Lechtken, D. Schooss, M.M. Kappes, F. Furche, Phys. Rev. A 77, 053202 (2008)

76. W. Huang, S. Bulusu, R. Pal, X.C. Zeng, L.S. Wang, ACS Nano 3(5), 1225 (2009)
77. R. Pal, L.M. Wang, W. Huang, L.S. Wang, X.C. Zeng, J. Chem. Phys. 134(5), 054306 (2011)

78. M.P. Johansson, I. Warnke, A. Le, F. Furche, J. Phys. Chem. C 118(50), 29370 (2014)

79. A. Tanwar, E. Fabiano, E.P. Trevisanutto, L. Chiodo, F. Della Sala, Eur. Phys. J. B 86(4), 161 (2013)

80. J. Li, X. Li, H.J. Zhai, L.S. Wang, Science 299(5608), 864 (2003)

81. J. Wang, J. Jellinek, J. Zhao, Z. Chen, R.B. King, P. von RaguéSchleyer, The Journal of Physical Chemistry A 109(41), 9265 (2005)

82. C.L. Cleveland, U. Landman, M.N. Shafigullin, P.W. Stephens, R.L. Whetten, Z. Phys. D: At., Mol. Clusters 40(1), 503 (1997)

83. C.L. Cleveland, U. Landman, T.G. Schaaff, M.N. Shafigullin, P.W. Stephens, R.L. Whetten, Phys. Rev. Lett. 79, 1873 (1997)

84. V. Petkov, Y. Peng, G. Williams, B. Huang, D. Tomalia, Y. Ren, Phys. Rev. B 72, 195402 (2005)

85. Z. Duan, Y. Li, J. Timoshenko, S.T. Chill, R.M. Anderson, D.F. Yancey, A.I. Frenkel, R.M. Crooks, G. Henkelman, Catal. Sci. Technol. 6, 6879 (2016)

86. Z.Y. Li, N.P. Young, M.D. Vece, S. Palomba, R.E. Palmer, A.L. Bleloch, B.C. Curley, R.L. Johnston, J. Jiang, J. Yuan, Nature 451, 46 (2008)

87. S.R. Plant, L. Cao, R.E. Palmer, Journal of the American Chemical Society 136(21), 7559 (2014), pMID: 24803386

88. J. Li, D. Yin, C. Chen, Q. Li, L. Lin, R. Sun, S. Huang, Z. Wang, Journal of Applied Physics $\mathbf{1 1 7}(8), 085303$ (2015)

89. Z.W. Wang, R.E. Palmer, Nano Letters 12(11), 5510 (2012)

90. R. Ouyang, Y. Xie, D.e. Jiang, Nanoscale 7, 14817 (2015)

91. C.D. Dong, X.G. Gong, The Journal of Chemical Physics 132(10), 104301 (2010)

92. D.M. Wells, G. Rossi, R. Ferrando, R.E. Palmer, Nanoscale 7, 6498 (2015)

93. C. Mottet, G. Tréglia, B. Legrand, Surf. Sci. 383 L719 (1997)

94. F. Baletto, R. Ferrando, A. Fortunelli, F. Montalenti, C. Mottet, J. Chem. Phys. 116, 3856 (2002)

95. T. Castro, R. Reifenberger, E. Choi, R.P. Andres, Phys. Rev. B 42, 8548 (1990)

96. I.L. Garzón, A. Posada-Amarillas, Phys. Rev. B 54, 11796 (1996)

97. H. Li, L. Li, A. Pedersen, Y. Gao, N. Khetrapal, H. Jónsson, X.C. Zeng, Nano Letters 15(1), 682 (2015), pMID: 25493586 
98. B.C. Curley, R.L. Johnston, N.P. Young, Z.Y. Li, M. Di Vece, R.E. Palmer, A.L. Bleloch, The Journal of Physical Chemistry C 111(48), 17846 (2007)

99. O.D. Häberlen, S.C. Chung, M. Stener, N. Rösch, J. Chem. Phys. 106(7), 5189 (1997)

100. H. Häkkinen, M. Moseler, O. Kostko, N. Morgner, M.A. Hoffmann, B. v. Issendorff, Phys. Rev. Lett. 93(9), 093401 (2004)

101. Y. Pei, N. Shao, Y. Gao, X.C. Zeng, ACS Nano 4(4), 2009 (2010), pMID: 20359242

102. I.L. Garzón, J.A. Reyes-Nava, J.I. RodríguezHernández, I. Sigal, M.R. Beltrán, K. Michaelian, Phys. Rev. B 66, 073403 (2002)

103. A.S. Barnard, L.A. Curtiss, ChemPhysChem 7(7), 1544 (2006)

104. S. Vergara, D.A. Lukes, M.W. Martynowycz, U. Santiago, G. Plascencia-Villa, S.C. Weiss, M.J. de la Cruz, D.M. Black, M.M. Alvarez, X. LópezLozano, C.O. Barnes, G. Lin, H.C. Weissker, R.L. Whetten, T. Gonen, M.J. Yacaman, G. Calero, The Journal of Physical Chemistry Letters 8(22), 5523 (2017), pMID: 29072840

105. M. Azubel, A.L. Koh, K. Koyasu, T. Tsukuda, R.D. Kornberg, ACS Nano 11(12), 11866 (2017), pMID: 29136369

106. T.R. Tero, S. Malola, B. Koncz, E. Pohjolainen, S. Lautala, S. Mustalahti, P. Permi, G. Groenhof, M. Pettersson, H. Häkkinen, ACS Nano 11(12), 11872 (2017), pMID: 29136363

107. E. Aprà, R. Ferrando, A. Fortunelli, Phys. Rev. B 73, 205414 (2006)

108. D. Schebarchov, F. Baletto, D.J. Wales, Nanoscale 10, 2004 (2018)

109. P. Koskinen, H. Häkkinen, G. Seifert, S. Sanna, T. Frauenheim, M. Moseler, New J. Phys. 8(1), 9 (2006)

110. P. Pyykko, Chem. Rev. 88(3), 563 (1988)

111. A. Fihey, C. Hettich, J. Touzeau, F. Maurel, A. Perrier, C. Köhler, B. Aradi, T. Frauenheim, J. Comput. Chem. 36(27), 2075 (2015)

112. L.F.L. Oliveira, N. Tarrat, J. Cuny, J. Morillo, D. Lemoine, F. Spiegelman, M. Rapacioli, J. Phys. Chem. A 120(42), 8469 (2016)

113. Y. Sugita, Y. Okamoto, Chem. Phys. Lett. 314(12), 141 (1999)

114. N. Tarrat, M. Rapacioli, J. Cuny, J. Morillo, J.L. Heully, F. Spiegelman, Comput. Theor. Chem. 1107, 102 (2017)

115. M. Rapacioli, N. Tarrat, F. Spiegelman, J. Phys. Chem. A 122(16), 4092 (2018)

116. M. Rapacioli, F. Spiegelman, N. Tarrat, Phys. Chem. Chem. Phys. 21, 24857 (2019)
117. A. Lechtken, C. Neiss, J. Stairs, D. Schooss, J. Chem. Phys. 129(15), 154304 (2008)

118. F. Furche, R. Ahlrichs, P. Weis, C. Jacob, S. Gilb, T. Bierweiler, M.M. Kappes, J. Chem. Phys. $\mathbf{1 1 7}(15), 6982$ (2002)

119. Z.W. Wang, R.E. Palmer, Nanoscale 4, 4947 (2012)

120. A. Lechtken, C. Neiss, J. Stairs, D. Schooss, J. Chem. Phys. 129(15), 154304 (2008)

121. D. Porezag, T. Frauenheim, T. Köhler, G. Seifert, R. Kaschner, Phys. Rev. B 51, 12947 (1995)

122. G. Seifert, D. Porezag, T. Frauenheim, Int. J. Quantum Chem. 58, 185 (1996)

123. M. Elstner, D. Porezag, G. Jungnickel, J. Elsner, M. Haugk, T. Frauenheim, S. Suhai, G. Seifert, Phys. Rev. B 58, 7260 (1998)

124. J. Cuny, N. Tarrat, F. Spiegelman, A. Huguenot, M. Rapacioli, J. Phys. Condens. Matter 30(30), 303001 (2018)

125. N. Tarrat, M. Rapacioli, F. Spiegelman, J. Chem. Phys. 148(20), 204308 (2018)

126. K.H. Hoffmann, P. Sibani, Phys. Rev. A 38, 4261 (1988)

127. J.O. Joswig, T. Lorenz, Z. Phys. Chem. 230, 1057 (2016)

128. D.J. Wales, M.A. Miller, T.R. Walsh, Nature 394, 758 (1998)

129. O.M. Becker, M. Karplus, J. Chem. Phys. 106, 1495 (1997)

130. A. Heuer, Phys. Rev. Lett. 78, 4051 (1997)

131. F. Calvo, A. Fortunelli, F. Negreiros, D.J. Wales, The Journal of Chemical Physics 139(11), 111102 (2013)

132. F.R. Negreiros, F. Taherkhani, G. Parsafar, A. Caro, A. Fortunelli, The Journal of Chemical Physics 137(19), 194302 (2012)

133. M. Asgari, F.R. Negreiros, L. Sementa, G. Barcaro, H. Behnejad, A. Fortunelli, The Journal of Chemical Physics 141(4), 041108 (2014) 


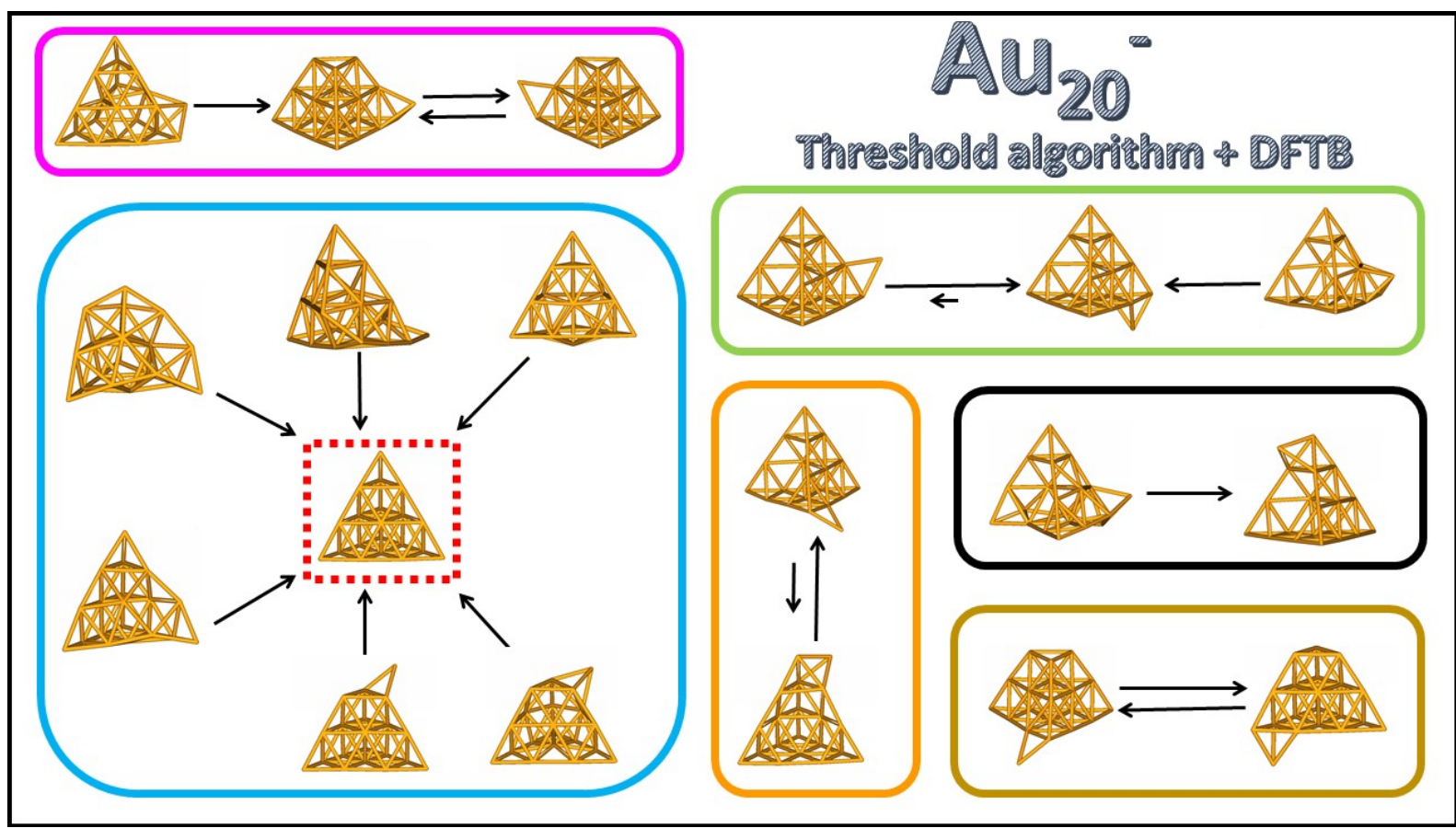

Fig. 6 TOC 